\title{
Categorical perception of stereoscopic stimuli
}

\author{
RICHARD D. HERRING and HAROLD P. BECHTOLDT \\ University of Iowa, Iowa City, Iowa 52242
}

\begin{abstract}
Two studies of the stereopsis model of Richards were conducted using a between-subjects balanced design and a repeated trials procedure rather than the within-subjects procedure of Richards. Measures of the accuracy of discrimination between classes of retinal disparity were defined by the method of successive intervals in place of the $d^{\prime}$ index of SDT. The results support the predictions (1) of symmetric discrimination functions for convergent and divergent stimuli, (2) of greater discrimination for stimuli of $\mathbf{4 5} \mathrm{min}$ of retinal disparity than for the stimuli of $15 \mathrm{~min}$ disparity, (3) of greater variability for the stimuli of greater disparity, and (4) of the use by nonanomalous subjects of at least two different processing mechanisms for stimuli of different disparity classes. The results do not support the prediction (1) of different processing mechanisms for foveal stimuli differing in figure-ground contrast or (2) of greater usefulness for monocular stimuli as a null disparity reference condition, as compared with binocular null disparity stimuli. Similar results were found for two stimulus shapes, a bar and a disk of equal area. Unreliability of subjects' response systems was suggested as a factor in the prediction of figure-ground contrast changes and as a factor in the classification of subjects as stereo anomalous. The pattern of accuracy of discrimination between classes of disparity stimuli also raises questions of the adequacy of the simple stereopsis model.
\end{abstract}

Whitman Richards $(1970,1971,1973,1975)$ has suggested that at least two separate neurophysiological mechanisms involving subcortical as well as cortical centers are used to process stereoscopic depth information for stimuli of convergent (crossed) and divergent (uncrossed) binocular disparities. Subjects exhibiting errors in judgment of depth of stimuli in one of the two disparity (crossed or uncrossed) categories are said to be stereo anomalous and to have a deficit in one of the postulated neural mechanisms; Richards (1975) estimates the relative frequency of anomalous subjects at about $30 \%$ of the population.

Since the direct evidence for this important hypothesis regarding mechanisms of stereopsis apparently comes from the laboratories of Richards or his associates (Foley, Applebaum, \& Richards, 1975; Foley \& Richards, 1974), in which relatively novel stimuli and procedures are used, the general applicability of the hypothesis needs further investigation. Two studies therefore were designed to provide evidence of the generality of the formulation proposed by Richards.

The interpretations offered by Richards as to the response properties of his two or more postu-

Experiment 1 constituted a portion of the requirements for the MA degree by $R$. D. Herring under the supervision of $H . P$. Bechtoldt. The editorial suggestions of Richard R. Hurtig are acknowledged with thanks. This investigation was conducted while the principal investigator was supported by the Visual Scholars Program, College of Education, University of lowa. The financial assistance of the Graduate College of the University of lowa for the computing funds is gratefully acknowledged. lated stereoscopic mechanisms are of concern (Foley, Applebaum, \& Richards, 1975; Richards, 1970, 1971, $1973,1975)$. In the first of two experiments, five predictions from Richards' model are of interest. These predictions are: (1) that convergent and divergent stimuli will be equally differentiated from a zero position with greater differentiation and greater variability for stimuli of $45 \mathrm{~min}$ binocular retinal disparity (BRD) as compared with stimuli of 15 min BRD; (2) that positive contrast stimuli will be differentiated more clearly than will negative contrast stimuli when foveal presentations are used; (3) that no effect of stimulus shape will be found; (4) that binocular null disparity stimuli will be less acceptable than monocular null disparity as reference stimuli; and (5) that at least two stereopsis processing mechanisms are utilized by nonanomalous cases.

Richards considers that the closer BRD approaches the nasal-temporal retinal overlap of $45 \mathrm{~min}$ of arc $(.75 \mathrm{deg})$, the higher is the correlation in activity (pooling of activity of the two mechanisms (Richards, 1975 , p. 366) and, apparently, the more accurate is the discrimination both from the null disparity and from the opposite class of stimuli. As the BRD approaches $45 \mathrm{~min}$ of arc, the number of excited elements increases, and so the variability of the neural activity will also increase (Foley, Applebaum, \& Richards, 1975; Richards, 1971, 1975). At levels of BRD greater than about $1 \mathrm{deg}$ of arc, other mechanisms apparently enter into the model.

The type of figure-ground contrast is considered an important stimulus property. Richards (1973, 
p. 862) suggests generally poor processing of negative contrast stimuli presented foveally. In addition, anomalous subjects are said to reverse their classification from, say, convergent anomalous to divergent anomalous with a change from stimuli with negative contrast to those with positive contrast and vice versa; the reversal effects will be confined to the "central region of vision."

Richards consistently uses a small vertical bar of about .25 by $2.0 \mathrm{deg}$ of visual angle. Such a vertical form extends to the parafoveal edges of the upper and lower retinal fields, for which some evidence of differential reactions to flashed stimuli has been reported under certain conditions (Breitmeyer, Battaglia, \& Bridge, 1977; Julesz, Breitmeyer, \& Kropfl, 1976; Payne, 1967). Pilot work using pairs of disk stimuli, separated vertically $5 \mathrm{deg}$, also gave some suggestive results. In view of the absence of any direct comment by Richards regarding the stimulus-shape and a possible parafoveal effect, it seemed reasonable to utilize as a second stimulus one that was confined to the fovea of equal area in the shape of a disk.

Richards has consistently used a monocular stimulus as the reference condition for his evaluation of convergent and divergent anomalous and stereoblind subjects; his preference for monocular stimuli as the reference condition has been justified largely on logical grounds. However, the recent work of Kaye (1978) indicates that monocular stimuli are associated with depth judgments varying in apparent distance with the eye stimulated. There is also some evidence that subjects with normal vision can tell which one of the two eyes is stimulated (Enoch, Goldmann, \& Sunga, 1969). For these reasons, the relative usefulness of monocular stimuli and of binocular stimuli of zero disparity as a reference condition warrants empirical investigation.

The distinctive feature of Richards' model is the postulation of two or even three different mechanisms to process the depth cues associated with convergent and divergent disparities and, possibly, the cues associated with zero disparity. The evidence for these postulated mechanisms develops from the difficulty exhibited by some subjects (anomalous or stereoblind) in discriminating between the depth cues provided by briefly flashed stimuli of the three different classes of binocular disparity.

Serious questions exist regarding the details of the procedures for dividing subjects into the classes of anomalous, stereoblind, or discriminating, on which these postulated mechanisms are based. The $\mathrm{d}^{\prime}$ indices of signal detection theory (SDT) regularly used by Richards require special rules for dichotomizing the three response categories and the series of stimulus disparity levels to form the two by two SDT data matrices.
Richards (1970, 1973), while combining data differently at different times, computes a $\mathrm{d}^{\prime}$ index derived from the three response categories of "in front," "on," and "in back" to describe the apparent location of each stimulus relative to the fixation point for each subject. In his more recent statement, Richards (1973, p. 857) has specified three separate $d^{\prime}$ indices. Apparently, the several stimuli within a type of disparity category are combined to form three levels of stimuli-convergent, divergent, and monocular.

One major change from the procedures used by Richards involves a new response measure developed from the method of successive intervals to express the accuracy of discrimination stimuli. Since the tworesponse analysis of SDT is a special case of Thurstone's method of successive intervals (MSI) expressed as a law of categorical judgment (Bock \& Jones, 1968; Green \& Swets, 1966; Jones, 1959; Lee, 1969; Torgerson, 1958) for which the maximum likelihood solution has been programmed (Schönemann \& Tucker, 1967), the MSI was selected as the basic method for scaling the categorical responses obtained from each subject. With the MSI method, no information is lost as a result of combining three or more stimulus levels or the frequencies in four or more response classes into two categories.

The MSI assumes that the internal, unobservable discrimination processes for each stimulus are separately, normally, and independently distributed around the mean of the stimulus discrimination processes from which the scale value of that stimulus is determined. The standard deviations of these distributions of internal processes are also estimated along with the boundaries of the response categories (Torgerson, 1958). Since the scaling can be accomplished for each subject separately, the variation of the scale values and the category boundaries over subjects within treatment conditions can be analyzed by standard normal statistical procedures; the analysis of the standard deviation data involves other distributional considerations. Although the programmed solution requires at least four response categories, a test of closeness of fit of the model to the data and maximum likelihood estimates of the three sets of model parameters for each subject make the MSI a desirable data analysis procedure.

In the second of two experiments, the consistency of performance of the subjects over time and over a change of figure-ground contrast in the stimuli is investigated. Although Richards regularly uses a within-subjects design, considerable evidence exists that indicates that perceptual processing of judgmental stimuli of ten is a function of the range and stimulus context of the experimental variable, especially in within-subjects designs (Erlebacher, 1977; Poulton, 
$1973,1979)$. Since the effects of the figure-ground contrast variable may well be influenced by biasing factors such as sequencing and stimulus change effects not represented in the stereopsis model of Richards, one effective method of control for such possible biasing factors is the between-subjects design.

The change from a within-subjects to a betweensubjects design is a second major change from the procedures used by Richards. The claims regarding the effects of a change in figure-ground contrast (Richards, 1973) can then be investigated by a systematic successive-trials design with a betweensubjects component. The objective of the second experiment is to evaluate both stability of performance of subjects over two successive test sessions of identical stimuli and stability, or lack thereof, in performance over two successive test sessions involving a change in figure-ground contrast.

Five response categories of depth judgments were provided in both experiments. The categories of clearly in front (CIF), in front (IF), at the same depth (S), in back (IB), and clearly in back (CIB) of the projection screen and fixation stimulus were explained to, and practiced by, the subjects prior to the experimental trials.

\section{GENERAL METHODS}

\section{Subjects}

The 20 volunteers of Experiment 1 and the nine volunteers of Experiment 2, all from an elementary psychology course, had uncorrected normal acuity and phorias as defined by performance on the Modified Orthorater (Bausch \& Lomb, Model 71-21-3102) or were corrected to normal vision by contact lenses; seven subjects from Experiment 1 and one from Experiment 2 were rejected for reasons of experimental error, equipment failure, or subject withdrawal.

\section{Experimental Design}

In Experiment 1, with one test session, the 20 subjects were pseudorandomly assigned with five cases per condition to four treatment conditions defined by stimulus shape (bar or disk) and by figure-ground contrast (negative contrast for black figures on white background or positive contrast for white figures on black backgrounds). Two within-subjects variables were type of disparity (crossed or convergent and uncrossed or divergent) and level of disparity (15 min or $45 \mathrm{~min}$ of binocular retinal disparity). A third within-subjects variable, whose effects were analyzed separately from those of the binocular stimuli, was the null or zero disparity condition, with two levels defined by the method of presentation as binocular or monocular (stimuli presented only to the left eye or to the right eye).

In Experiment 2, with two test sessions, 10 subjects were assigned randomly to two experimental groups with five cases per group. One of the subjects failed to complete the experiment. The two groups corresponded to the two types of figure-ground contrast for the disk stimuli of Experiment 1.

\section{Apparatus}

The stimuli were projected onto a Polacoat rear projection screen by a modified Compco stereo slide projector (Model 500) equipped with $300-\mathrm{W}$ projęction bulbs. For binocular presentations, the two projection fields were polarized at $90 \mathrm{deg}$ with respect to each other by stock Polaroid filters; monocular pre- sentations were created by polarizing each field in the same direction, using filters fashioned in our laboratories.

A tachistoscope shutter system with a three-channel timing device was designed for the stereo projector. Two of the three timer channels pulsed two high-speed dc motors (Airborne Accessories Corporation Model E1600 M13-1), which turned rotary shutters in front of each projection lens. The positions of the shutters were controlled by photosensors and light sources.

The fixation stimuli were presented by a Kodak Carousel projector Model 800 . The third channel of the timer activated a rotary solenoid (Ledex 1-1075-036), which controlled a Wollensak Alphax shutter in front of the projection lens.

The rear projection screen was located $39 \mathrm{~cm}$ in front of the subjects, who viewed the stimuli on the screen through Polaroid filters attached to a chin- and headrest.

\section{Stimuli}

The projected bar- and disk-shaped stimuli subtended $.5 \mathrm{deg}$ square visual angle (VA) on the Polacoat screen. The images of the bars were $.25 \mathrm{deg}$ VA wide $(1.66 \mathrm{~mm})$ and $2 \mathrm{deg}$ VA high $(13.3 \mathrm{~mm})$, while the images of the disks had a radius of $.39 \mathrm{deg}$ VA $(2.65 \mathrm{~mm})$. The initial black cardboard models of the stimulus figures on white backgrounds were photographed with $35-\mathrm{mm}$ Kodalith film negatives and printed on fine-grain positives. Identical pairs of $35-\mathrm{mm}$ film frames were placed in stereo-slide mounts, with Kodalith negatives providing the positive contrast condition (white figures on black grounds); the positive film provided the negative contrast condition (black targets on white grounds). The contrast levels for the two classes of stimuli were approximately equal and were estimated at better than $80 \%$.

Five stimulus locations, corresponding to five depth levels used in each of the four treatment conditions, were controlled manually by the experimenter through template markings on the rear side of an opaque shield to the rear of the projection screen. These locations provided the 15 and $45 \mathrm{~min}$ of binocular disparity for the convergent (in front of the screen) and the divergent (in back of the screen) disparities and for the zero disparity (on the screen).

The averaged figure-ground luminances of the designs measured by a photometer (Soligar Spot Sensor II) through the subject's polarizing filters on the chinrest were $.2 \mathrm{~cd} / \mathrm{m}^{2}$ for the light stimuli on dark grounds and $2.4 \mathrm{~cd} / \mathrm{m}^{2}$ for the dark stimuli on the light grounds.

The fixation stimulus consisted of four small, red, wedgeshaped figures in a diamond design of $5.28 \mathrm{deg}$ VA (35 mm) horizontally and $3.76 \mathrm{deg}$ VA $(25 \mathrm{~mm})$ vertically. The figures of the fixation design all fell outside the target stimulus area. Separate fixation slides and an iris diaphragm were used to match the averaged fixation design luminance to the averaged luminance of the target stimuli. The fixation stimulus was on the screen except when the target stimuli were presented.

\section{Procedure}

In the first of two or three sessions, the subjects were briefed, consent was obtained, and visual acuity and phorias were measured (not stereopsis). In the second (or third) session, 109 or more practice and test trials were administered, with each trial involving a ready signal, instructions to blink and fixate in the middle of the fixation design, and presentation of a stimulus design (one of the five binocular or two monocular) $1 \mathrm{sec}$ later for a duration of $80 \mathrm{msec}$ to which the subject responded with one of the five response categories: clearly in front (CIF), in front (IF), at the same depth (S), in back (IB), and clearly in back (CIB) of the projection screen.

Initially, 13 practice trials were presented with feedback in three blocks of trials; the first block of five trials utilized stimulus durations of $480 \mathrm{msec}$, and the second block, durations of $180 \mathrm{msec}$. If a subject made more than two incorrect responses in a block, another block was presented at that speed. The final three practice trials were presented at $80 \mathrm{msec}$. 
Following the practice trials, the test stimuli were presented randomly in two successive blocks of 48 trials each without feedback at $80 \mathrm{msec}$ duration. Each block included eight trials at each of the five binocular locations and four trials of each of the two monocular zero disparity conditions. For purposes of analysis, the responses to the two monocular stimuli were combined into a single score, with the result that each of six stimulus conditions was presented eight times in each of the two blocks, for a total of 16 presentations each.

The procedures of Experiment 1 were used for the first test session of Experiment 2, with different groups of subjects receiving the negative contrast and positive contrast slides. On the second test session of Experiment 2, both sets of slides were presented, with the first set a repetition of the slides used in the first test session. The second set of slides for each group was that assigned to the other group in the first test session; the change was a "reversal shift" type of manipulation for the figure-ground contrast variable.

\section{EXPERIMENT 1}

\section{Data Analysis}

A scaled score was defined as the mean scale value from the MSI maximum likelihood procedure (Schönemann \& Tucker, 1967), with the computed negative values (arbitrarily) representing distances toward the subject or in front of the screen, and positive values representing distances behind or beyond the screen, away from the subject. A zero point was defined for each subject as the centroid of the person's scale values; as a result of the symmetric design of the set of stimuli, this zero point falls on or near the projection screen. In order to eliminate the negative values for the purpose of presenting some data graphically, 5 was added to all means, with the result that the origin or projection screen value was 5 rather than 0 .

Since Richards (1973) specifies a set of three d' values to be used to define the stereoanomalous condition, these $d^{\prime}$ values were calculated for each subject for the purpose of comparing the classification of subjects by two procedures. The major differences in the classification results reflected the fine-grain analyses of the MSI method as contrasted with the broad categorization of the d' procedure.

Since the MSI procedure provides maximum likelihood estimates of the standard deviations of the response distributions of each stimulus for each subject, as well as the estimates of the means of the distributions, a series of directional $t$ tests developed for the definitions of the stereoanomalous conditions. Each set of four directional $t$ tests evaluates the discrimination between two pairs of stimuli, each pair representing one type of disparity; the comparisons are between convergent (C) and null disparity ( 0 ) stimuli, between convergent $(C)$ and divergent (D) stimuli, and between divergent (D) and null disparity (0) stimuli. A failure to discriminate between two sets of stimuli is indicated by three or four $t$-test failures $(t>-.9)$, while a marginal condition is indicated by two t-test failures. The classification of convergent or divergent anomalous is based on the failures to discriminate $C$ from 0 and $D$ from 0 , respectively. A stereoblind classification requires failure in all three sets of comparisons in the MSI procedure.

\section{Results}

The mean values of the basic frequency data are presented in Table 1; the possible range of frequency values is from 0 to 16 . The mean values in Table 1 are based on five cases, with a different sample represented in each of the four successive rows of the table corresponding to each level of amount of disparity within a type of disparity. The type of dis- parity and amount of disparity are within-subjects variables. The modal mean frequency in each set of five response categories is italicized to provide a visual indication of the generally close relationship of the judgments of the subject to the stimulus variables in Experiment 1.

Scaled scores. The scaled scores were sensitive to the effects of the independent variables. The fourvariable ANOVA of the results for the pairs of convergent and divergent disparity stimuli indicates a main effect of type of disparity $[F(1,16)=59.67$, $\mathrm{p}<.05]$ and three interactions involving the two classes of disparity. These three interactions were Amount of Disparity by Type of Disparity $[\mathrm{F}(1,16)$ $=27.28, \mathrm{p}<.05]$, Type of Disparity by FigureGround Contrast $[\mathrm{F}(1,16)=13.61, \mathrm{p}<.05]$, and Amount of Disparity by Type of Disparity by FigureGround Contrast $[\mathrm{F}(1,16)=6.21, \mathrm{p}<.05]$.

The main effect and three interactions with type of disparity can be discussed in terms of the data for the triple interaction shown in Figure 1; the values plotted are the mean scaled scores plus 5.0. The main effect of type of disparity is shown by the mean values greater than 5.0 (responses of behind the screen) for the four stimuli with divergent binocular disparity and by the mean values less than 5.0 (responses of in front of the screen) for the four stimuli with convergent disparity.

The interaction of Type of Disparity by Amount of Disparity is created by the more extreme values of the means for the 45-min-disparity stimuli compared with the mean values for the 15-min-disparity stimuli. The Type of Disparity by Figure-Ground Contrast interaction developed from the greater absolute magnitudes of the mean scaled across for the negative contrast (black on white) stimuli than for the mean scaled scores for the positive contrast (white on black) stimuli. Simple effects were analyzed as single contrasts using the error term for the interaction. The simple effects of the four functions shown in Figure 1 indicate reliable trends $(p<.05)$ for both types of disparity at both levels of disparity and for figure-ground contrast at all of the four treatment combinations, except for the effect of figure-ground contrast at $\mathbf{1 5}$ min disparity for convergent stimuli.

Since the trend lines of Figure 1 appeared to be symmetrically placed around the zero point of 5.0 as Richards predicted, a symmetry analysis of the data was made using a four variable ANOVA after multiplying the convergent scaled scores for each subject by $(-1,0)$. The results are consistent with the hypothesis of symmetry for convergent and divergent stimuli, since there was no main or interaction effect with type of disparity.

For the two classes of null disparity stimuli, the scaled scores indicated an effect only for type of disparity $[\mathrm{F}(1,16)=18.68, \mathrm{p}<.05]$. The binocular 
Table 1

Mean Frequency of Responses in Each of Five Response Categories to Stimuli Varying in Type of Disparity, Amount of Disparity, Figure-Ground Contrast, and Shape for Four Groups of Five Subjects Each

\begin{tabular}{|c|c|c|c|c|c|c|c|c|}
\hline \multicolumn{2}{|c|}{ Disparity } & \multirow{2}{*}{$\begin{array}{c}\text { Figure-Ground } \\
\text { Contrast }\end{array}$} & \multirow[b]{2}{*}{ Shape } & \multicolumn{5}{|c|}{ Response Category* } \\
\hline Type & Amount & & & CIF & IF & $\mathbf{S}$ & IB & CIB \\
\hline \multirow{2}{*}{ Convergent } & \multirow{2}{*}{45} & Positive & $\begin{array}{l}\text { Bar } \\
\text { Disk }\end{array}$ & $\begin{array}{r}8.0 \\
10.4\end{array}$ & $\begin{array}{l}4.2 \\
2.6\end{array}$ & $\begin{array}{r}1.0 \\
.2\end{array}$ & $\begin{array}{l}.8 \\
.8\end{array}$ & $\begin{array}{l}2.0 \\
2.0\end{array}$ \\
\hline & & Negative & $\begin{array}{l}\text { Bar } \\
\text { Disk }\end{array}$ & $\begin{array}{r}8.8 \\
13.6\end{array}$ & $\begin{array}{r}5.2 \\
.8\end{array}$ & $\begin{array}{r}1.0 \\
.0\end{array}$ & $\begin{array}{l}.4 \\
.2\end{array}$ & $\begin{array}{r}.6 \\
1.4\end{array}$ \\
\hline \multirow{2}{*}{ Convergent } & \multirow{2}{*}{15} & Positive & $\begin{array}{l}\text { Bar } \\
\text { Disk }\end{array}$ & $\begin{array}{l}3.8 \\
3.6\end{array}$ & $\begin{array}{l}7.8 \\
8.6\end{array}$ & $\begin{array}{r}.6 \\
2.4\end{array}$ & $\begin{array}{l}3.0 \\
1.4\end{array}$ & $\begin{array}{l}.8 \\
.0\end{array}$ \\
\hline & & Negative & $\begin{array}{l}\text { Bar } \\
\text { Disk }\end{array}$ & $\begin{array}{l}3.0 \\
4.2\end{array}$ & $\begin{array}{l}9.2 \\
9.0\end{array}$ & $\begin{array}{l}2.8 \\
1.2\end{array}$ & $\begin{array}{r}1.0 \\
.6\end{array}$ & $\begin{array}{r}.0 \\
1.0\end{array}$ \\
\hline \multirow{2}{*}{ Binocular Null } & \multirow{2}{*}{0} & Positive & $\begin{array}{l}\text { Bar } \\
\text { Disk }\end{array}$ & $\begin{array}{r}.2 \\
1.2\end{array}$ & $\begin{array}{l}3.0 \\
5.0\end{array}$ & $\begin{array}{r}11.6 \\
7.6\end{array}$ & $\begin{array}{l}1.2 \\
2.2\end{array}$ & $\begin{array}{l}.0 \\
.0\end{array}$ \\
\hline & & Negative & $\begin{array}{l}\text { Bar } \\
\text { Disk }\end{array}$ & $\begin{array}{r}.6 \\
1.0\end{array}$ & $\begin{array}{l}7.0 \\
6.0\end{array}$ & $\begin{array}{l}7.0 \\
8.6\end{array}$ & $\begin{array}{r}1.4 \\
.4\end{array}$ & $\begin{array}{l}.0 \\
.0\end{array}$ \\
\hline \multirow{2}{*}{ Monocular Null } & \multirow{2}{*}{0} & Positive & $\begin{array}{l}\text { Bar } \\
\text { Disk }\end{array}$ & $\begin{array}{l}.6 \\
.8\end{array}$ & $\begin{array}{l}1.6 \\
3.6\end{array}$ & $\begin{array}{l}8.2 \\
5.8\end{array}$ & $\begin{array}{l}4.2 \\
4.0\end{array}$ & $\begin{array}{l}1.4 \\
1.8\end{array}$ \\
\hline & & Negative & $\begin{array}{l}\text { Bar } \\
\text { Disk }\end{array}$ & $\begin{array}{l}.0 \\
.0\end{array}$ & $\begin{array}{r}1.0 \\
.8\end{array}$ & $\begin{array}{l}12.4 \\
13.0\end{array}$ & $\begin{array}{l}2.4 \\
1.6\end{array}$ & $\begin{array}{l}.2 \\
.6\end{array}$ \\
\hline \multirow{2}{*}{ Divergent } & \multirow{2}{*}{15} & Positive & $\begin{array}{l}\text { Bar } \\
\text { Disk }\end{array}$ & $\begin{array}{l}.4 \\
.6\end{array}$ & $\begin{array}{l}2.2 \\
4.8\end{array}$ & $\begin{array}{l}2.8 \\
4.8\end{array}$ & $\begin{array}{l}8.4 \\
5.6\end{array}$ & $\begin{array}{r}2.2 \\
.2\end{array}$ \\
\hline & & Negative & $\begin{array}{l}\text { Bar } \\
\text { Disk }\end{array}$ & $\begin{array}{r}.0 \\
1.0\end{array}$ & $\begin{array}{r}.4 \\
1.2\end{array}$ & $\begin{array}{l}1.6 \\
2.0\end{array}$ & $\begin{array}{r}11.2 \\
9.2\end{array}$ & $\begin{array}{l}2.8 \\
2.6\end{array}$ \\
\hline \multirow{2}{*}{ Divergent } & \multirow{2}{*}{45} & Positive & $\begin{array}{l}\text { Bar } \\
\text { Disk }\end{array}$ & $\begin{array}{l}3.6 \\
1.6\end{array}$ & $\begin{array}{l}1.6 \\
1.6\end{array}$ & $\begin{array}{l}1.6 \\
1.4\end{array}$ & $\begin{array}{l}3.4 \\
5.8\end{array}$ & $\begin{array}{l}5.8 \\
5.6\end{array}$ \\
\hline & & Negative & $\begin{array}{l}\text { Bar } \\
\text { Disk }\end{array}$ & $\begin{array}{l}.0 \\
.8\end{array}$ & $\begin{array}{l}.2 \\
.4\end{array}$ & $\begin{array}{l}.2 \\
.6\end{array}$ & $\begin{array}{l}3.6 \\
5.4\end{array}$ & $\begin{array}{r}12.0 \\
8.8\end{array}$ \\
\hline
\end{tabular}

Note-Amount of disparity is given in minutes of arc. The four groups were defined by two classes of figure-ground contrast and two shapes; type of disparity and amount of disparity were within-subjects variables. Positive figure-ground contrast designs had a white figure on a black ground; negative contrast designs had a black figure on a white ground. "See text for response code.
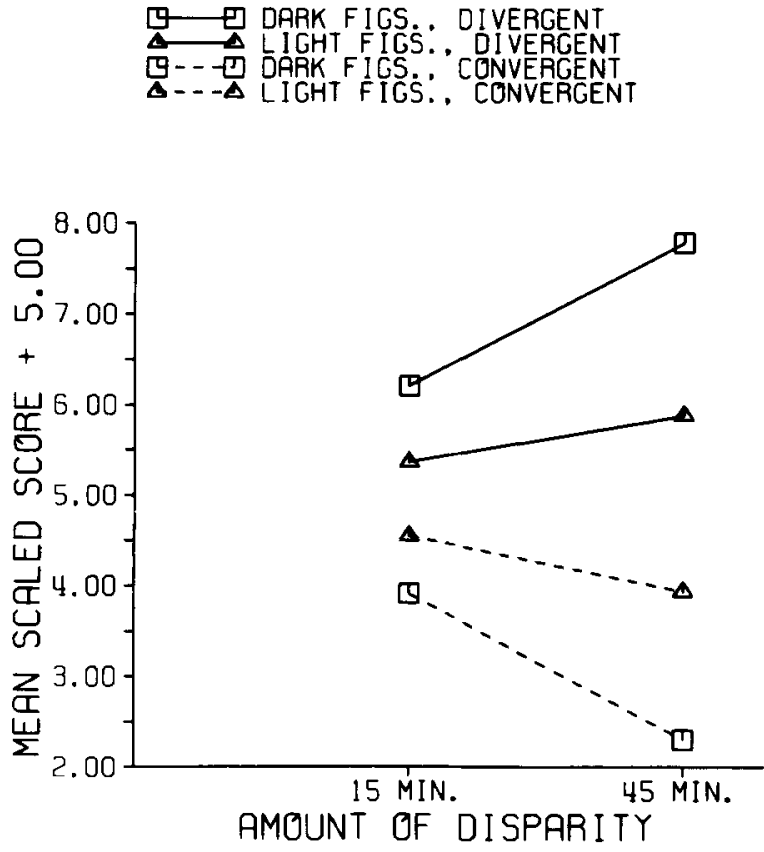

Figure 1. Means of scaled scores plus live of depth judgments to stimuli varying in figure-ground contrast, type of disparity, and amount of disparity. zero disparity stimuli with mean scaled scores of -.20 were judged somewhat in front of the fixation point, while the monocular stimuli with mean scaled scores of .25 were judged somewhat behind the fixation point.

Standard deviations of discriminative processes. The estimated standard deviations from the MSI Procedure provide data relevant to the hypothesized greater neural activity associated with stimuli of greater retinal disparity (Foley, Applebaum, \& Richards, 1975). The average standard deviations of the 45- and 15-min-disparity stimuli for each of the four groups of subjects ranged from 1.26 to 1.40 for the 45-min stimuli, with a mean of 1.36 , and from .44 to .90 for the 15 -min stimuli, with a mean of .66. The Friedman matched groups procedure with a $\chi_{\mathrm{r}}^{2}$ test statistic was used to analyze the standard deviation data. The significant $\chi_{\mathrm{r}}^{2}$ value of $\mathbf{1 7 . 7 6}$ for $3 \mathrm{df}, \mathrm{p}<.05$, for the total sample of 20 subjects is consistent with the suggestion that the standard deviations for the 45-min stimuli exceeded those for the 15-min stimuli.

For the null disparity stimuli, the overall sign test was not significant. The overall mean standard deviations were .365 and .452 for the binocular and monocular stimuli, respectively. 
Categorization of subjects. The $d^{\prime}$ procedure of Richards (1973) was used as the basic procedure. According to this procedure, 12 of the 20 subjects were classed as discriminators or "normal"; 8 of the subjects were indicated as stereoanomalous. Five of the 8 cases were divergent anomalous, 2 were convergent anomalous, and 1 was marginally stereoblind. For the MSI procedure, 12 subjects were classed as discriminators and 8 as stereoanomalous as in the $\mathrm{d}^{\prime}$ procedure. Four of the 8 cases were classed as divergent anomalous, 2 as convergent anomalous, and 2 as marginally stereoblind. The proportion of anomalous subjects was found to be .40 , a value consistent, for a sample of 20 subjects, with the $30 \%$ suggested by Richards.

Variations in these classification results are created by the deviation of the observed frequencies in the data matrices from the simple diagonal pattern of frequencies that would be predicted from a linear order (of apparent distances) of two or three stimuli. Comparison of the original data with the MSI values suggests, for example, that only one of the six stimuli is discriminated from the remaining five by some subjects.

\section{Discussion}

The results of this study clearly support most of the predictions associated with the model of stereopsis suggested by Richards. As predicted, the mean values provided evidence of symmetrical discrimination functions for convergent and divergent stimuli, with greater discrimination for the stimuli of $45 \mathrm{~min}$ of retinal disparity than for the stimuli of $15 \mathrm{~min}$ of disparity. Greater variability, as predicted by Richards, for the stimuli of greater disparity was also observed. Since MSI tends to allocate larger variances and wider categories to the extremes of the stimulus set, this evidence of greater variability for the 45-min stimuli is not unequivocal. However, this objection may not be critical, since, for 9 of the 20 subjects, the two largest standard deviations were not for the 45-min stimuli.

Evidence in support of the postulation of at least two stereopsis processing mechanisms was provided by the classification of four or five subjects as divergent anomalous, two or three as convergent anomalous, and one or two as stereoblind. In addition, the estimation by Richards of about $30 \%$ as the relative frequency of anomalous cases was also supported. The variation among classification decisions for one subject was attributable, in part, to the presence of the largest standard deviation for the monocular stimulus in the set of six stimuli. The presence of consistent sets of responses indicating shifts in the linear ordering of the stimuli, if confirmed, will require a modification of the details of the basic model.
The general rejection on a priori grounds of binocular null disparity stimuli in favor of monocular stimuli as reference stimuli is not supported by the data. There is little, if any, evidence of any general difference favoring either one of the two null disparity stimuli, either in the analysis of mean scale values or in the analyses of standard deviations.

The predicted greater discrimination for the stimuli of positive figure-ground contrast (white figures on black) was not obtained; in fact both the convergent and divergent negative contrast stimuli were more clearly differentiated at both levels of disparity than were the positive contrast stimuli. This discrepancy of the observed results from those predicted by Richards may be related to the level of contrast used; in the current study, the contrast levels were as nearly equal as we could achieve, while Richards has emphasized the lack of figure-ground contrast in his negative contrast stimuli, although he has also said the contrast level for the dark bars was about $90 \%$. We saw nothing in our negative contrast stimuli that would correspond to the report by Richards of a type of rivalry and appearance of sheen for his dark bar stimuli.

The difference in results also may be due to a difference in experimental procedures; the betweensubjects design was used with only one contrast level (negative or positive stimuli) presented to each subject, since we wished to deal directly with that figure-ground contrast variable. Richards used both classes of stimuli with a within-subjects design, but blocked his positive and negative stimuli in the Kodak projector tray for each subject. The result would be marked variations in general brightness level from one stimulus type to the other within a session in the procedure used by Richards; in such procedures, the negative contrast effect would involve a brightness or glare recovery function at least at the point of change from positive to negative contrast stimuli.

The shape variable proved to be of little importance beyond evidence of generality of the relationship for foveal stimuli. Although the difference in performance associated with the bar and disk stimuli was not significant, the data indicated that the disk stimuli were at least as acceptable as the bar stimuli. There was no evidence of any differential effect associated with the parafoveal extension of the bar stimuli.

\section{EXPERIMENT 2}

While the first experiment provided considerable support for several predictions from the stereopsis model, at least two issues needed further consideration. First, the figure-ground contrast effects of Experiment 1 were not consistent with the inter- 
pretations of fered by Richards; and, second, the consistency of classification of subjects over at least two sets of occasions and a change in figureground contrast has not been considered. A small repeated trials study involving two groups of subjects was designed to provide data relevant to these two issues. The procedures and disk stimuli of Experment 1 were used in Experiment 2.

\section{Data Analysis}

The scaled scores and the classification procedures of Experiment 1 were computed for each subject for each of the three sessions. The data for the two groups receiving disk stimuli in Experiment 1 were used, together with those of the first set of trials in Experiment 2, to evaluate the extent of the sampling variations over semesters for samples of five cases each. Since these calculations bias the results toward achieving a replication of Experiment 1 while providing evidence of stability of the results, a separate analysis of the data of the first set of trials (Session 1) of Experiment 2 was also conducted. A confidence interval analysis was also computed for the comparison of means between experiments, as suggested for small samples by Tversky and Kahneman (1971). The Friedman test for matched groups was used to analyze the ordering of the standard deviations for the nonnull disparity stimuli, and the sign test was used with the data from the two null disparity stimuli.

Average within-subjects product-moment correlations summed over the six stimuli of each trial were used to evaluate the consistency of responses over the three pairs of trials for the nine cases completing both sessions. The evaluation of the consistency of classification of subjects required another procedure. There were three pairs of discriminations to be made in each trial; these were C vs. 0, C vs. D, and D vs. 0, as described in Experiment 1. Three levels of each discrimination were defined as in Experiment 1, based on the MSI t tests, as successful, marginal, or failure. The frequency of agreement in accuracy of discrimination for each pair of trials was computed as the basic data.

\section{Results and Discussion}

Scaled scores. The analyses of the scaled scores for the nonnull disparity stimuli indicated a partial replication of the effects of Experiment 1. There was a main effect of type of disparity $[F(1,16)=$ $31.94, p<.05]$ and an interaction of Type of Disparity by Amount of Disparity $[\mathrm{F}(1,16)=17.04$, $p<.05$ ]; the evaluation of this interaction must involve consideration of two further effects. The mean scaled scores from Experiment 1 exceeded those from Experiment 2 in the Amount of Disparity by Experiment interaction $[F(1,16)=5.11, p<.05]$ and in the Type of Disparity by Amount of Disparity by Experiment interaction $[\mathrm{F}(1,16)=5.72$, $\mathrm{p}<.05]$.

A simple effect analysis of this last interaction indicated that the mean scaled scores of both the convergent and divergent disparity conditions for Experiment 1 exceeded the mean values of Experiment 2 at 45 min disparity $(p<.05)$ but not at 15 min disparity; the trend lines for the Experiment 1 data, but not for the Experiment 2 data, located the $45-\mathrm{min}$ stimuli as more extreme than the 15-min stimuli. A significant interaction of Amount of Disparity by Figure-Ground Contrast $[\mathrm{F}(1,16)=$ $6.23, p<.05$ ] involved a crossover of two trend lines, which, however, referred to means within the range of -.11 to +.08 ; the error term used in this comparison was extremely small.

A failure to replicate is suggested by the absence of a triple interaction of Type of Disparity by Amount of Disparity by Figure-Ground Contrast, as diagrammed for Experiment 1 in Figure 1. In both experiments, the means were ordered in the same way; however, the negative contrast means in Experiment 2 were only slightly more extreme than the positive contrast means. The $95 \%$ confidence inter$\mathrm{val}$ analysis of the differences in means for the contrasts in Figure 1 indicated overlapping regions for all pairs of corresponding means in the two experiments, except for the difference between the means of the 45-min stimuli of positive contrast and convergent disparity; in that exceptional comparison, the Experiment 1 value of -1.52 was far more extreme than was the Experiment 2 value of -.40 .

Further evidence of the variability of the effect of the figure-ground contrast variable is provided by a separate analysis of the scaled score data from Session 1 of Experiment 2 alone. As in Experiment 1 , two significant effects in the analysis of the one set of Experiment 2 data were the type of disparity $[F(1,8)=24.30, p<.05]$ and an interaction of Amount of Disparity by Type of Disparity $[F(1,8)=10.05, p<.05]$. The other interactions significant in Experiment 1 were, at best, only marginally significant $(\mathrm{p}<.10)$ in Experiment 2 .

For the null disparity stimuli in the combined analysis, the significant effect for type of stimuli $[F(1,16)=8.63, p<.05]$ arises from the binocular stimuli being judged slightly in front of the fixation point with a mean of -.09 and the monocular stimuli judged slightly behind the screen with a mean scaled score of .16, as was the case in Experiment 1.

Standard deviations of discriminative processes. The average standard deviations for the 45- and 15-min-disparity stimuli in the first set of trials were 1.41 and .91 , respectively. The Friedman test statistic applied to the four standard deviations from each of the 10 subjects was significant $\left[x_{r}^{2}(3)=\right.$ 8.04, $\mathrm{p}<.05$ ]. As in Experiment 1, for several of the subjects, the standard deviations for the 15-min stimuli were ranked as the largest. The variation in standard deviations for the null disparity stimuli was not significant by the sign test around an average value of .38 , with seven of the 10 cases showing larger standard deviations for the monocular stimuli.

Reliability estimates of scaled scores. The average within-subjects correlations constituted a homogeneous set, with three chi-square values of $13.71,13.80$, and 15.57 for $8 \mathrm{df}, \mathrm{p}>.01$. The average correlation 
between the six scores for the first two trials was $.86[t(7)=4.46, p<.01]$; these trials represented stability of performance over a $24-\mathrm{h}$ interval. The average correlation for the comparisons of the scores of Trials 1 and 2 with those of Trial 3, in which the figure-ground reversal took place, were also significant at $\alpha=.05$. The correlation between the Trial 1 and Trial 3 values was .73 $[\mathrm{t}(7)=2.83, \mathrm{p}<.05]$ and, for Trials 2 and 3 , the value was $.69[\mathrm{t}(7)=2.52$, $p<.05]$. The three correlations were clearly positive and of comparable magnitude.

Categorization of subjects. As in the presentation of the Experiment 1 results, the $d^{\prime}$ procedure of Richards was the basic procedure, with the MSI results considered in relation to the $d^{\prime}$ procedure. According to the $\mathrm{d}^{\prime}$ classification rules applied to the data from Session 1, 4 of the 10 cases were anomalous; 1 was classed as convergent anomalous, 1 as divergent anomalous, and 2 as stereoblind. The $40 \%$ figure agrees with the results of Experiment 1 and the predictions of Richards.

As was the case in Experiment 1, differences in classifying subjects were indicated by the two procedures. Only three anomalous cases $(30 \%)$ were indicated by the MSI procedure. Of the two cases classed as stereoblind by the $d^{\prime}$ rules, one was classed as blind but the other as divergent anomalous by MSI; the two subjects classed by $\mathrm{d}^{\prime}$ as convergent and divergent anomalous were, at worst, only marginally anomalous by the MSI procedure, and a $\mathrm{d}^{\prime}$ marginal subject was classed as stereoblind by MSI. As in the Experiment 1 results, these differences were related to shifts in the ordering of the stimuli from the linear order associated with the type and amount of disparity and variations in the standard deviations of each stimulus. For some subjects, only one of the six stimuli was discriminated from the others, and that stimulus might not be at the extremes of the physically defined order.

Consistency of classification. Since the classification of subjects is based on their accuracy of discrimination between pairs of classes of stimuli, the general stability of such discriminations according to the MSI t-test procedure over pairs of trials is the matter of interest. Six of the nine subjects $(67 \%)$ in seven comparisons and seven of the nine $(78 \%)$ in the other two comparisons exhibited the same accuracy of discrimination over the three trials; four of the subjects exhibited the same pattern of discrimination on every trial. There is no evidence of any systematic change in the accuracy of discrimination between the stimulus classes C vs. 0 , $C$ vs. $D$, and D vs. 0 over changes in figure-ground contrast or over a 24 -h period.

There were, however, changes in classification for three of the nine subjects. One subject shifted from divergent anomalous in the first trial to stereoblind in the second and to convergent anomalous in the third trial. One other subject rated as stereoblind in the first session improved in the second and third sets of trials to marginal or to discriminator classification. One subject with a discriminator rating on the first two sets of trials fell to a divergent rating on the third set.

\section{GENERAL DISCUSSION}

As noted earlier, the results offer general support for the broad outline of the model proposed by Richards. As predicted, symmetrical functions were found for convergent and divergent disparity stimuli. with the 45-min stimuli located farther from the fixation point than were the 15-min stimuli; the average variability for the $45-\mathrm{min}$ stimuli also was larger than that of the 15 -min stimuli for about $60 \%$ of the subjects. A more adequate test of the hypothesized greater variability can be made with the method of successive intervals by including a wider range of stimuli extending beyond $45 \mathrm{~min}$ of BRD as well as more stimuli in the range of 0 to $45 \mathrm{~min}$.

The hypothesis of three different mechanisms for processing convergent and divergent disparities and for processing null disparity stimuli is also supported by the generally consistent evidence from the two methods of data analysis-the method of successive intervals and the signal detection theory methods. Subjects were found whose responses indicated confusion of convergent and of divergent stimuli with null disparity stimuli, but no confusion between the convergent and divergent stimuli; other subjects showed confusion of convergent with divergent stimuli, but differentiation of these stimuli from the null disparity stimuli. Using the specified critical cutoff values guided by Richards' published work, we reported from $30 \%$ to $40 \%$ stereo anomalous cases, depending on the specific computing procedure applied to the two samples of cases. These values are certainly in line with the $30 \%$ suggested by Richards.

The results of the MSI procedure raise serious questions both about the adequacy of the SDT procedure of Richards, in which no evaluation of the $C$ vs. $D$ discrimination is made, and about the assumption of constant standard deviations used in the $\mathrm{d}^{\prime}$ procedure. Marked variation in the standard deviations of some stimuli of all types of disparity were found for some subjects. Differentiation of only one stimulus from the other five was also seen, with the discriminated stimulus not being one of the extreme 45-min values.

The simple linear model involving the three disparity classes of $C, 0$, and D stimuli, with separate mechanisms for each class of stimuli, is parsimonious and heuristically valuable. However, until further 
work is carried out with the MSI procedure to list the frequency and types of failures of the model, the rather common acceptance of the classification of subjects as anomalous or stereoblind seems premature. In part, basic unreliability of a subjects' response system is a plausible alternative to the typological emphasis of that classification for many subjects.

It seems likely that a variety of effects having relevance to the model will arise as depth judgments are obtained with a variety of stimulus designs. While the predictions regarding the figure-ground contrast variable were not confirmed, the introduction of other stimuli into the visual field, as in the adjacency principle (Gogel, 1969), should prove to be an important factor, possibly requiring modification of the model. The work of Kaye (1978), using textured and untextured backgrounds, is another example. It seems unlikely that the fundamental stereopsis mechanisms would be delimited by the use of an isolated, briefly flashed static or dynamic stimulus.

The present study also raises the old question of the most useful experimental design for perceptual problems. Although the use of a few highly practiced subjects in a within-subjects design is common practice in perceptual experiments, it is an empirical question in each case whether the results are generalizable to new samples, to new stimulus arrangements, and to investigations using between-subjects designs. Considerable evidence of the desired generalization of the results of Richards' model from within-subjects to between-subjects designs was obtained; perhaps the figure-ground variable is one that does not generalize.

\section{REFERENCES}

Bock, R. D., \& Jones, L. V. The measurement and prediction of judgment and choice. San Francisco: Holden-Day, 1968.

Breitmeyer, B., Battaglia, F., \& Bridge, J. Existence and implications of a tilted binocular disparity space. Perception, $1977,6,161-164$.

Enoch, J., Goldman, H., \& Sunga, R. The ability to distinguish which eye was stimulated by light. Journal of Investigative Ophthalmology, 1969, 8, 317.331.
Erlebacker, A. Design and analysis of experiments contrasting the within- and between-subjects manipulation of the experimental variable. Psychological Bulletin, 1977, 84, 212-219.

Foley, J. M., Applebaum, T. H., \& Richards, W. A. Stereopsis with large disparities: Discrimination and depth magnitude. Vision Research, 1975, 15, 417-421.

Foley, J. M., \& Rich ARDS, W. Improvement in stereoanomaly with practice. American Journal of Optometry and Physiological Optics, 1974, 51, 935-938.

Gogel, W. C. Equidistance effects in visual fields. American Journal of Psychology, 1969, 82, 342-349.

GreEN, D. M., \& Swets, J. A. Signal detection theory and psychophysics. New York: Wiley, 1966.

HAYS, W. L. Statistics for psychologists. New York: Holt, Rinehart \& Winston, 1963.

Jones, L. V. Some invariant findings under the method of successive intervals. American Journal of Psychology, 1959, 72, 210-220.

Julesz, B., Bhe itmeyer, B., \& Kropfl, W. Binocular disparitydependent upper-lower hemifield anisotropy and left-right hemifield isotropy as revealed by dynamic random-dot stereograms. Perception, 1976, 5, 129-141.

KAYE, M. Stereopsis without binocular correlation. Vision Research, 1978, 18, 1013-1022.

LEE, W. Relationships between Thurstone category scaling and signal detection theory. Psychological Bulletin, 1969, 71, 101-107.

PAyne, W. H. Visual reaction times on a circle about the fovea. Science, 1967, 155, 481-482.

Poulton, E. C. Unwanted range effects from using within-subject experimental designs. Psychological Bulletin, 1973, 80, 113-121.

Poulton, E. C. Models for biases in judging sensory magnitudes. Psychological Bulletin, 1979, 86, 777-803.

Richards, W. Stereopsis and stereoblindness. Experimental Brain Research, 1970, 10, 380-388.

Rich ARDS, W. Anomalous stereoscopic depth perception. Journal of the Optical Society of America, 1971, 61, 410-414.

Richards, W. Reversal in stereo discrimination by contrast reversal. American Journal of Optometry and Archives of American Academy of Optometry, 1973, 50, 853-862.

Richards, W. Visual space perception. In E. Carterette \& $\mathbf{M}$. Friedman (Eds.), Handbook of perception: Seeing (Vol. 5). New York: Academic Press, 1975.

Richards, W., \& Foley, J. Interhemispheric processing of binocular disparity. Journal of the Optical Society of America, $1971,61,419-421$.

Schönemann, P. H., \& Tucker, L. R. A maximum-likelihood solution for the method of successive intervals allowing for unequal stimulus dispersions. Psychometrika, 1967, 32, 403-417.

TORGERSON, W. S. Theory and methods of scaling. New York: Wiley, 1958.

TVersky, A., \& Kahneman, D. Belief in the law of small numbers. Psychological Bulletin, 1971, 76, 105-110.

(Received for publication June 17, 1980; accepted September 10, 1980.) 